\title{
METODOLOGÍA ABP: HABILIDADES DE AUTONOMÍA Y TRABAJO COLABORATIVO EN ESTUDIANTES DE BACHILLERATO
}

\author{
Angie Ximena Valles Ramírez \\ ax.vallesramirez@gmail.com \\ https://orcid.org/0000-0002-5159-2806 \\ Patricia Covarrubias-Papahiu \\ papahiu@unam.mx \\ https://orcid.org/0000-0001-9855-3399 \\ Universidad Nacional Autónoma de México (UNAM). México.
}

Recibido: 2020-07-17; Aceptado: 2020-11-22

\begin{abstract}
Resumen
Las altas tasas de reprobación y baja eficiencia terminal, influyen negativamente en el desarrollo del estudiante y el desempeño de la institución educativa, por ello se han realizado esfuerzos para asegurar la permanencia escolar. En concordancia, el ABP es una estrategia de enseñanza que ha mostrado promover la motivación y habilidades que inciden en el aprendizaje significativo de contenidos curriculares. El objetivo de la investigación fue promover la autonomía y el trabajo colaborativo en estudiantes de bachillerato, implementando la metodología ABP con herramientas de apoyo virtual para el aprendizaje de contenidos de psicología. Se realizó un estudio cuasiexperimental con diseño pre-test- post-test, utilizando un grupo experimental y uno control. Los resultados señalan mejoras significativas en el aprendizaje de contenidos y el trabajo colaborativo. Se propone la implementación de la metodología ABP con herramientas de apoyo virtual en el nivel medio superior para lograr aprendizajes significativos y promover el aprendizaje colaborativo.
\end{abstract}

Palabras clave: Aprendizaje basado en problemas, autonomía, trabajo colaborativo.

\section{PBL METHODOLOGY: AUTONOMY AND COLLABORATIVE WORK DEVELOPMENT IN SENIOR HIGH SCHOOL STUDENTS}

Abstract

High academic failure and low promotion rates reflect the negative development of students and scholar institutions; therefore, several actions have been made to ensure students retention. Accordingly, PBL is a teaching approach that enables students to enhance motivation and skills related to course content meaningful learning. A quasi-experimental, two group pretest-posttest design was conducted to improve senior high school student's autonomy and collaborative work in psychology class by using a PBL methodology and virtual tools. The results showed significant improvements in content learning and collaborative work. Researchers suggest the implementation of the PBL methodology and virtual support tools to achieve meaningful learning and collaborative work in high school students.

Keywords: Problem based learning, autonomy, collaborative work. 


\section{METODOLOGIA ABP: HABILIDADES DE AUTONOMIA E TRABALHO COLABORATIVO EM ALUNOS DO ENSINO MÉDIO \\ Resumo}

As altas taxas de reprovação e a baixa eficiência terminal influenciam negativamente no desenvolvimento do aluno e no desempenho da instituição de ensino, portanto, esforços têm sido feitos para garantir a retenção escolar. Nesse sentido, a Aprendizagem Baseada em Problemas - ABP, é uma estratégia de ensino que tem demonstrado promover motivação e habilidades que influenciam na aprendizagem significativa dos conteúdos curriculares. $\mathrm{O}$ objetivo da pesquisa foi promover a autonomia e o trabalho colaborativo em alunos do ensino médio, implementando a metodologia $\mathrm{ABP}$ com ferramentas virtuais de apoio à aprendizagem de conteúdos de psicologia. Um estudo quase experimental foi realizado com um desenho préteste-pós-teste, utilizando um grupo experimental e um grupo controle. Os resultados indicam melhorias significativas na aprendizagem do conteúdo e no trabalho colaborativo. A implementação da metodologia ABP é proposta com ferramentas de suporte virtual no nível secundário superior para alcançar uma aprendizagem significativa e promover a aprendizagem colaborativa.

Palavras-chave: Aprendizagem baseada em problemas, autonomia, trabalho colaborativo.

\section{Introducción}

La metodología del Aprendizaje Basado en Problemas (ABP) tuvo gran auge después de ser empleada en el currículo de la Universidad de McMaster en Canadá, pues fue gestada desde una postura constructivista que pretendía mejorar la calidad educativa y apoyar la formación integral del estudiante por medio de la autogestión de aprendizajes, la socialización del aprendizaje individual y la solución de problemas contextuales creados por el docente con base en determinados objetivos de aprendizaje (Barrows, 1992).

La mayor bondad del ABP radica en que es el estudiante quien busca los contenidos que considera necesarios para dar solución a los problemas planteados, logrando adquirir y desarrollar habilidades no consideradas en métodos tradicionales, tales como, capacidad investigativa, aprendizaje autónomo, trabajo colaborativo y pensamiento crítico (Clemens, Tapia y Olivares, 2015; Biggs, 2010; Boud y Feletti, 1997; Duch, Groh y Allen, 2001; HmeloSilver y Ferrari, 1997; Wilkerson y Gijselaers, 1996).

Estas habilidades permiten que el estudiante interiorice su proceso de aprendizaje generando motivación y un aprendizaje significativo (Branda, 2009). Esto, eventualmente evitará el desfase entre las expectativas de los sistemas educativos y los resultados del estudiante que participa en ellos, expresado a través de las bajas tasas de reprobación o los niveles de eficiencia terminal (Martínez, 2009). 
La preocupación por reducir el fracaso escolar y facilitar el aprendizaje de contenidos en la Educación Media Superior (EMS) deriva de informes de organismos como la OCDE (2014) e informes como el de la Encuesta Nacional de Deserción en Educación Media Superior (ENDEMS, 2012) y entidades públicas, los cuales señalan que en México apenas el 53\% de jóvenes de quince a diecinueve se matriculan en este nivel. Advierten que la poca motivación en los estudios y las bajas por reprobación son factores que inciden en el fracaso escolar y que, como advierten tales organismos, es imprescindible darles solución (Mondragón y Fuentes, 2016).

La motivación y la creación de un ambiente escolar positivo son condiciones que mejoran la construcción del conocimiento y el desarrollo de habilidades necesarias para una formación integral, que pueden implementarse como formas de trabajo cuando una institución educativa requiere mejorar el rendimiento escolar de sus estudiantes (Gómez y Mei, 2007).

El Colegio de Ciencias y Humanidades $(\mathrm{CCH})$ de la Universidad Nacional Autónoma de México (UNAM), ante el panorama descrito, propone realizar investigaciones con orientaciones a la elaboración de estrategias para la experiencia docente que contribuyan al enriquecimiento del programa y la promoción de acciones orientadas a fortalecer la responsabilidad y autonomía de los estudiantes (ENCCH, 2017).

La metodología ABP puede ser una alternativa que permite desarrollar habilidades de aprendizaje, que incidan en los índices de reprobación y deserción escolar (Fernández y Duarte, 2013). Si bien, en la revisión documental no se encontraron propuestas metodológicas detalladas desde el ABP en EMS para impartir la materia de psicología en México, existen estudios en otras áreas del conocimiento que han contribuido al mejoramiento de los procesos de aprendizaje aún y cuando no detallan, ni muestran al lector la propuesta desarrollada (Pantoja y Covarrubias, 2013; Ríos, Urrutia y Contreras, 2013)

La presente investigación tuvo como objetivo promover la autonomía y el trabajo colaborativo en estudiantes de $5^{\circ}$ semestre del $\mathrm{CCH}$ Vallejo mediante la aplicación del Aprendizaje Basado en Problemas (ABP) como metodología que contempla elementos adicionales a la sola presentación de un problema. En particular, se diseñó y aplicó esta propuesta para la enseñanza del tema Escuelas psicológicas de la asignatura de Psicología, que se imparte en quinto semestre del $\mathrm{CCH}$. Aunque por coherencia epistémica, no se puede pretender realizar un protocolo específico a manera de prescripción para llevar a cabo sesiones 
de enseñanza desde la metodología ABP, en esta investigación se buscó mostrar y reflejar los fundamentos teóricos y metodológicos utilizados en el proceso de enseñanza aprendizaje.

\section{Fundamentos teóricos y metodológicos del ABP}

La metodología ABP posee sus supuestos teóricos desde el constructivismo pedagógico para comprender la fuente psicopedagógica que da sustento teórico al constructivismo, es necesario exponer las diferentes teorías de forma resumida (Carretero, 2009).

Por ejemplo, Piaget propone que la producción del conocimiento se da a partir de las estructuras cognitivas de asimilación y acomodación, las cuales participan en el proceso de aprendizaje y permiten usar esquemas y dar sentido a algo nuevo o cambiar esquemas para poder responder a las demandas del entorno, respectivamente. Además plantea que el desequilibrio cognitivo es fundamental en los procesos de aprendizaje pues permite al individuo transitar hacia la asimilación y la acomodación (Coll, Palacios y Marchesi, 2001).

Ausubel, Novak y Hanesian (2012), proponen al aprendizaje significativo como aquel que se construye a través de ideas de anclaje o conocimientos previos, que dan significado a los nuevos, permitiendo que el aprendiz los convierta en parte de sus esquemas cognitivos y se genere un proceso más sólido de aprendizaje. Y Vygotsky enfatiza el origen social del aprendizaje y su dependencia de la comunicación e interacción entre el contexto socio-histórico y el aprendiz, de tal modo que en el proceso educativo, el aprendizaje ocurre colaborativamente a través del diálogo y donde el docente funge como mediador y co-constructor interactivo de conocimientos (Álvarez y Del Rio, 1990).

Contemplar las anteriores posturas dentro de la metodología ABP, permite evitar el reduccionismo biológico, social o cognitivo del ser humano comprendiendo el proceso aprendizaje-enseñanza como una red compleja que se teje de forma sólida al contemplar las características individuales de cada uno de sus protagonistas.

El ABP, originado en Canadá ha sido utilizado como metodología y estrategia de aprendizaje (Dueñas, 2001). Desde sus orígenes, el ABP contempla un grupo reducido de estudiantes, un problema a resolver con ayuda del docente o facilitador del conocimiento, la participación activa de los estudiantes en el proceso de aprendizaje y el descubrimiento de los saberes necesarios para alcanzar los objetivos y resolver el problema inicial (William y Gallagher, 1993). 
Lo anterior, teniendo claridad en que el problema no solo tiene como fin la construcción de una solución por parte del estudiante, sino que es el escenario que permite la ocurrencia del aprendizaje por descubrimiento. En este transitar, el estudiante es capaz de desarrollar los aprendizajes esperados por el docente.

En consecuencia, la discusión destaca como un elemento que estimula a los estudiantes durante el proceso, en un nivel intelectual, al explorar diferentes perspectivas, analizar su discurso y reestructurar sus esquemas con ayuda de los esquemas de los otros protagonistas del aula. En un nivel social, construyendo a su vez la importancia de la democracia, el respeto al otro, la identidad de grupo y el trabajo colaborativo. Y en un nivel afectivo, permitiendo que el estudiante perciba el apoyo del grupo en sus procesos de construcción personal, lo cual incrementa la motivación al comprender la importancia de sus aportaciones, la tolerancia a la frustración y la pertenencia a un grupo (Vizcarro y Juárez, 2008).

En este sentido, el papel del docente consiste en facilitar la construcción de conocimiento y el cumplimiento de objetivos por medio de su experticia, dando pautas al estudiante para vincular información pertinente y guiando sus procesos de búsqueda de información para resolver el problema planteado. Además es responsable de generar un ambiente escolar positivo, diseñar la planeación de las sesiones y fomentar la interacción en el grupo (Morales y Landa, 2004).

En cuanto al papel del estudiante, consiste en asumir un rol activo en el proceso de construcción del aprendizaje, participando, debatiendo y asumiendo la responsabilidad que implica colaborar con sus ideas para que los otros construyan el conocimiento (Sola, 2005).

Respecto al problema, Albanese y Mitchell (1993) proponen tres variables básicas para su formulación: a) Relevancia, referida a que el problema debe importar al estudiante y vincularlo intelectual y emocionalmente. b) Cobertura, dirigida a que el problema sea claro, coherente y se relacione con los contenidos a tratar. c) Complejidad, la cual implica que el problema a resolver no tiene una única solución y guía a la interdisciplinariedad.

Para la resolución del problema y el desarrollo de las sesiones, existen diferentes pasos propuestos para el desarrollo de la metodología ABP, como los siete pasos de Maastrich para grupos de hasta 40 estudiantes (Schmit, 1983), las cuatro fases al estilo de Hong Kong para grupos de hasta 60 estudiantes (Lai y Chu, 2000), el modelo de Alcalá para grupos de hasta 130 estudiantes (Prieto, Barbarroja, Reyes, Monserrat, Díaz, Villarroel y Álvarez, 2006). 
En el presente trabajo se retoman los postulados de Morales y Landa (2004), quienes proponen que se debe transitar por ocho fases de desarrollo del ABP en el proceso de enseñanzaaprendizaje: 1) leer y analizar el escenario del problema, 2) realizar una lluvia de ideas, 3) enlistar lo que se conoce, 4) enlistar lo que se desconoce, 5) listar lo necesario para resolver el problema, 6) definir el problema, 7) obtener información y 8) obtener resultados. Si bien esta propuesta no contempla la cantidad de estudiantes que pueden ser partícipes del proceso, si contempla las interacciones docente-estudiante necesarias en el ABP.

Los roles del docente y estudiante, así como las condiciones que requiere el ABP, permiten que el estudiante desarrolle autonomía, autorregulación, trabajo colaborativo, pensamiento crítico, mayor motivación por los contenidos temáticos a abordar y la capacidad para autoevaluarse, contemplando los tiempos dedicados a la solución del problema y la coherencia de su propuesta (Rué, 2009; González, Martin, Carbonero y Landa, 2013).

La utilización de TIC's propicia la organización de contenidos y facilita material didáctico al estudiante (Figura 1). Particularmente en el ABP, promueve la colaboración, el almacenamiento de archivos y facilita las tareas de cada una de las fases del ABP (Benítez, Cruces, De Haro y Sarrión, 2010; Romero, Jara, Marín, Millán y Carrillo, 2009). 


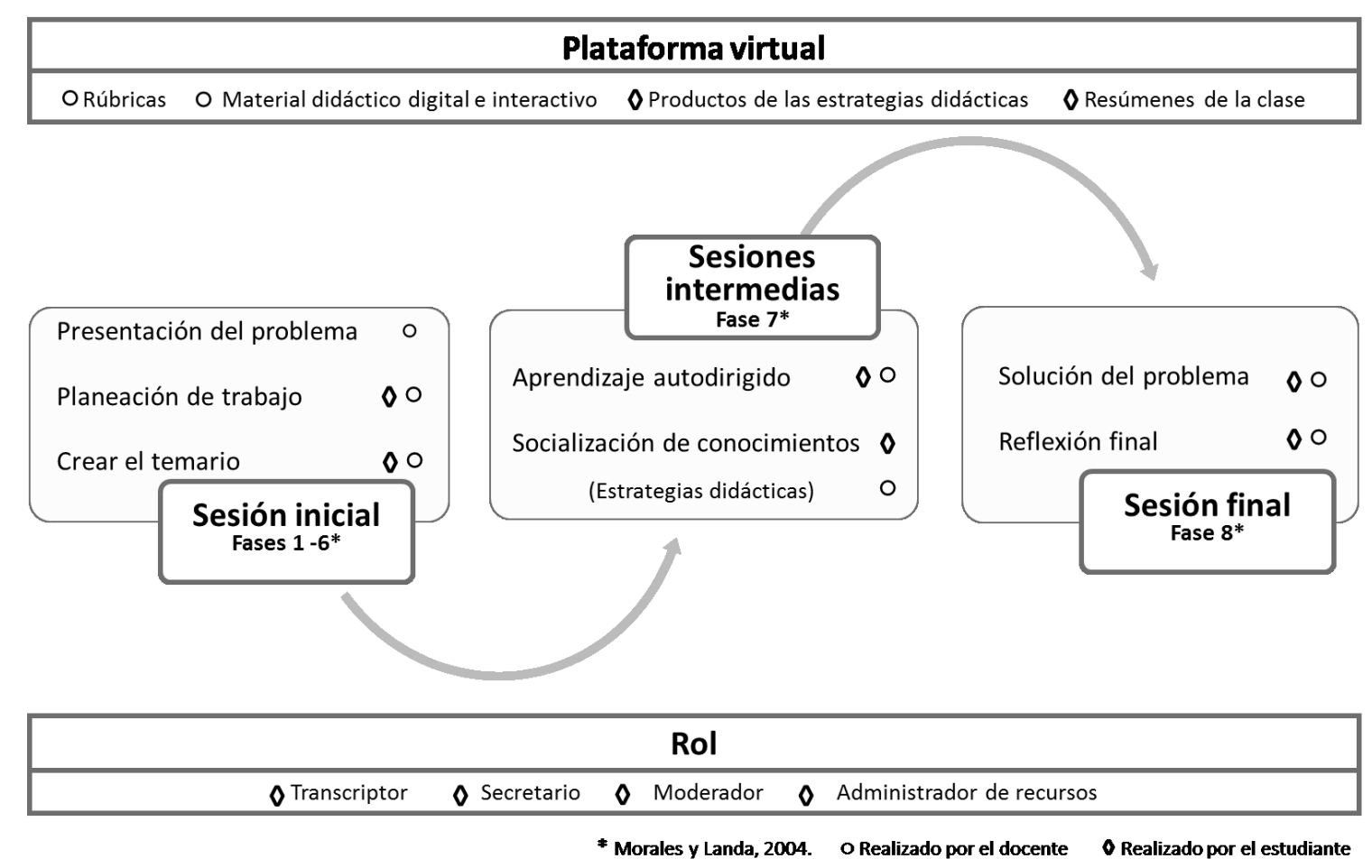

Figura 1. Fundamentación metodológica del ABP.

Fuente: Elaboración propia.

\section{Autonomía intelectual (AI)}

Autonomía intelectual, autonomía o autogestión del aprendizaje, aprendizaje autónomo y aprender a aprender, son conceptos que refieren la capacidad de apropiarse del conocimiento (Flores y Meléndez, 2017; Solórzano-Mendoza, 2017; Brockbank y McGill, 2002).

Para Benson (2001), la AI es un constructo multidimensional que incluye factores metacognitivos, sociales y afectivos, los cuales refieren a su vez, categorías que permiten abordar la complejidad del constructo, definido como la capacidad del estudiante para responsabilizarse de su aprendizaje en términos del control de sus propios procesos cognitivos participantes en la autorregulación eficaz del aprendizaje.

Además, incluye aspectos psicológicos como el desarrollo de habilidades metacognitivas resultado del papel activo del estudiante. Entre las operaciones que el estudiante desarrolla para gestionar su aprendizaje (habilidades cognitivas), están la planificación, referida a la organización de la tarea de aprendizaje, determinar cómo desarrollarla, establecer los objetivos (O’Malley y Chamot, 1990; Cyr, 1996 y Oxford, 1990). 
La gestión de la atención, refiere al mantenimiento del interés en la clase al evitar distractores, la autogestión, que implica identificar las condiciones facilitadoras del aprendizaje y su puesta en práctica. Finalmente, el automonitoreo, referido a corregir constantemente sus actividades mientras las desarrolla y la autoevaluación, orientada a valorar avances y capacidades para realizar tareas y usar estrategias (Benson, 2001).

La autonomía intelectual se encuentra vinculada a la autonomía moral, la cual incluye el factor social, contemplando comportamientos del estudiante como hacer preguntas al profesor o compañeros para aclarar o verificar si su comprensión de un concepto es correcta, cooperar con otros, compartiendo información y reconociéndolos como pares e incluyéndolos en la resolución de problemas, ser empático y comprender las diferencias de pensamientos, sentimientos, perspectivas y culturas (Benson, 2001).

Finalmente, el factor afectivo, definido como el control que se debe tener para reforzar la confianza en sí mismo, motivarse y ver los errores como oportunidades de aprendizaje, categorías como bajar la ansiedad, referida a la utilización de técnicas de respiración o relajación, autoanimarse, que implica decirse frases positivas, tomar riesgos, no temer equivocarse, y reconocer su buen desempeño, y control de emociones, que consiste en compartir sentimientos (Benson, 2001).

\section{Trabajo colaborativo}

El trabajo colaborativo tiene sus referentes conceptuales en la teoría constructivista e implica el trabajo en equipo de los estudiantes como principales actores del proceso de aprendizaje, y consiste en construir el conocimiento desde la dialéctica y el modelo dialógico (Díaz-Barriga, 2005).

Existen diferentes beneficios al hacer uso del trabajo colaborativo como el fomento de la metacognición, la práctica de habilidades sociales, permitir la sensación de control sobre la tarea, permitir el reconocimiento de diferentes perspectivas y crear un ambiente escolar positivo (Kolloffel, Eysink y Jong, 2011).

En la presente investigación conceptualizamos el trabajo colaborativo como la habilidad para trabajar en un contexto de aprendizaje desde la igualdad, la mutualidad, la distribución de responsabilidades, la planificación conjunta, el intercambio de roles y la bidireccionalidad (Díaz-Barriga, 2005). 


\section{Metodología de la investigación}

Se realizó la investigación en un ambiente educativo natural, bajo un diseño cuasiexperimental, con un esquema de línea pre-test-intervención-post-test (A-B-A), con dos grupos intactos (Grupo experimental y Grupo control).

\section{Objetivos}

El objetivo general de esta investigación consistió en promover la autonomía y el trabajo colaborativo en estudiantes de $5^{\circ}$ semestre del $\mathrm{CCH}$ Vallejo mediante la aplicación del Aprendizaje Basado en Problemas (ABP) como metodología didáctica. Para ello se establecieron como objetivos específicos:

1) Diseñar una estrategia didáctica basada en la metodología del ABP para el aprendizaje del tema "Escuelas psicológicas" de la asignatura de Psicología de $5^{\circ}$ semestre del $\mathrm{CCH}$.

2) Promover la construcción de conocimientos en el tema La diversidad de la psicología de la materia de Psicología I.

\section{Participantes}

El grupo experimental, en el que se implementó la metodología $\mathrm{ABP}$, estuvo constituido por 29 estudiantes que cursaban la materia de psicología en el CCH-Vallejo de 16 a 22 años. Se realizaron nueve sesiones presenciales de dos horas cada una, y dos horas adicionales desarrolladas en un aula virtual, para cumplir un total de 20 horas de dedicación al tema $L a$ diversidad de la psicología. La propuesta de intervención incluyó material didáctico convencional, audiovisual y digital.

El grupo control estuvo conformado por 30 estudiantes que también cursaban la materia de psicología en el CCH-Azcapotzalco, con un rango de edades de los 16 a 21 años. En este grupo no se aplicó el ABP, se revisó el mismo tema La diversidad de la psicología, pero con las estrategias didácticas con las que habitualmente trabaja la maestra titular de la materia introducción al tema, exposiciones, lecturas de tarea, conformación de equipos de discusión, presentación de ejemplos cotidianos, mapas conceptuales y resúmenes-. Se realizaron diez 
sesiones de dos horas para cumplir 20 horas de estudio. Se incluyó material didáctico convencional y audiovisual.

El horario en que se llevaron a cabo las sesiones fue de 7:00 am a 9:00 am, en ambos grupos.

Instrumentos

Para evaluar los efectos de la aplicación del ABP, se aplicaron varios instrumentos de evaluación respetando las características de la metodología ABP.

\section{Encuesta de Autonomía Intelectual}

La encuesta, validada por Restrepo (2018) se compone de 55 reactivos de los cuales 32 corresponden a estrategias metacognitivas que incluyen planificación, gestión de la atención, autogestión, automonitoreo y autoevaluación; otros 13 reactivos que corresponden a estrategias sociales, que incluyen la habilidad para hacer preguntas, cooperar con otros y sentir empatía. Y finalmente 10 reactivos que conciernen a la evaluación de estrategias afectivas como bajar la ansiedad, autoanimarse y controlar las emociones.

Esta prueba fue adaptada y validada con población mexicana. El valor de coeficiente alfa de Cronbach fue de 0.84 indicando una alta consistencia interna y un buen índice de confiabilidad. Mediante el análisis factorial se encontraron 3 dimensiones, que correspondieron a las tres estrategias consideradas por Benson (2001), arriba descritas.

Este instrumento se aplicó tanto al grupo experimental como al control, antes y después de haber revisado el tema.

\section{Rúbrica de trabajo colaborativo}

Se elaboró con cinco criterios: participación del grupo, responsabilidad compartida, carga equitativa del trabajo, roles en el grupo y habilidades interpersonales. Estos criterios fueron establecidos teniendo en cuenta la revisión conceptual sobre el trabajo colaborativo de Kolloffel, Eysink y Jong (2011) y la implementación del ABP.

Este instrumento solo fue aplicado al grupo experimental, en cada una de las nueve sesiones presenciales, ya que en la metodología utilizada por la profesora del grupo control, no se pretendía implementar el trabajo colaborativo. 


\section{Cuestionario de conocimientos sobre "La diversidad de la psicología"}

Se diseñó un cuestionario de 10 ítems para evaluar conocimientos sobre la diversidad de la psicología. Teniendo en cuenta la lógica del ABP, estuvo compuesto de problemas donde el estudiante debía demostrar la capacidad que tiene para contextualizar el conocimiento aprendido a lo largo del curso. Se utilizaron ítems del tipo relación de casos o problemas contextuales con elección múltiple y completar mapas, para evaluar aprendizajes conceptuales y procedimentales contemplados en el programa de la materia de Psicología I del CCH. Se realizó un pilotaje con 40 estudiantes de psicología para determinar validez de contenido. El programa Test Analysis Program 4.2 arrojó índices de discriminación mayores a 0.2 , asegurando un buen índice de dificultad del cuestionario.

Este instrumento se aplicó como pre-test (Fase A), tanto al grupo experimental como al control, para conocer los conocimientos previos con los que contaban los estudiantes, y después de haber revisado el tema (Fase B), como posevaluación (Fase A) para conocer los conocimientos adquiridos.

\section{Procedimiento y recogida de datos}

Con base en los aprendizajes esperados en la asignatura, se diseñó una planeación didáctica y se procedió iniciar la intervención en el aula. Cada sesión inició con la presentación del problema a los estudiantes.

"En una colonia existe una banda que se dedica a robar casas. Ya existen 6 miembros y 2 de estos se dedican a reclutar adolescentes en el parque. La comunidad se da cuenta y desea acabar con la situación, su casa es una de las afectadas. El Jefe de la junta vecinal decide convocar una reunión con los ciudadanos y después de un mes capturan al líder de la banda, que es menor de edad. Las autoridades estatales, solicitan la ayuda de un grupo de psicólogos pues no saben qué hacer para que el líder de la banda no vuelva a delinquir".

A) Como psicólogo, ¿qué enfoque le quedaría mejor respecto a la propuesta inicial que

dio? B) Si usted fuera el adolescente del problema, ¿A qué psicólogo iría?” (Preguntas realizadas en la primera sesión)

De acuerdo a este problema, los estudiantes realizaban una primera lluvia de ideas que los llevara a indagar sobre sus conocimientos previos y los nuevos conocimientos que debían construir. 
Durante la primera hora se realizaba aprendizaje guiado autodirigido -coordinados por el estudiante con el rol de recursos y el docente-, en la siguiente hora se realizaban diferentes estrategias didácticas - texto teatral narrativo, cuadro sinóptico, dialogo igualitario, analogías, resolución de problemas y debates-, que permitían compartir el conocimiento generado en la primera hora -coordinadas por los estudiantes bajo los roles de moderador y secretario-

Paralelamente al desarrollo de las sesiones se construyó un aula virtual en la plataforma Moodle. En ésta se subían los resúmenes de clase elaborados por los estudiantes, así como videos, infografias, referencias y juegos relacionados con los contenidos revisados en clase.

Para el análisis de datos se utilizó el programa estadístico SPSS26.

\section{Resultados}

\section{Encuesta de Autonomía Intelectual}

Para asegurar el grado de consistencia y la estabilidad de las puntuaciones de la encuesta, se calculó el índice de confiabilidad obteniendo una alpha de cronbach de 0,87 para la preprueba y 0,81 en el post-test, el cual nos permite afirmar que la validez de esta encuesta fue del $87 \%$ en el pre-test y del $81 \%$ en el post-test por lo que es un instrumento que permite medir la autonomía.

Al ser una encuesta estadísticamente confiable, se analizaron los datos descriptivos.

Según la Figura 2, se puede concluir que los dos grupos obtuvieron características similares en los niveles de autonomía en el pre-test y el post-test; reflejando un nivel alto de autonomía (122-143) y homogeneidad en los grupos para el pre-test $\left(\sigma_{1}=8,88, \sigma_{2}=14,74\right)$ y el post-test $\left(\sigma_{1}=9,9 \sigma_{2}=9,7\right)$. 


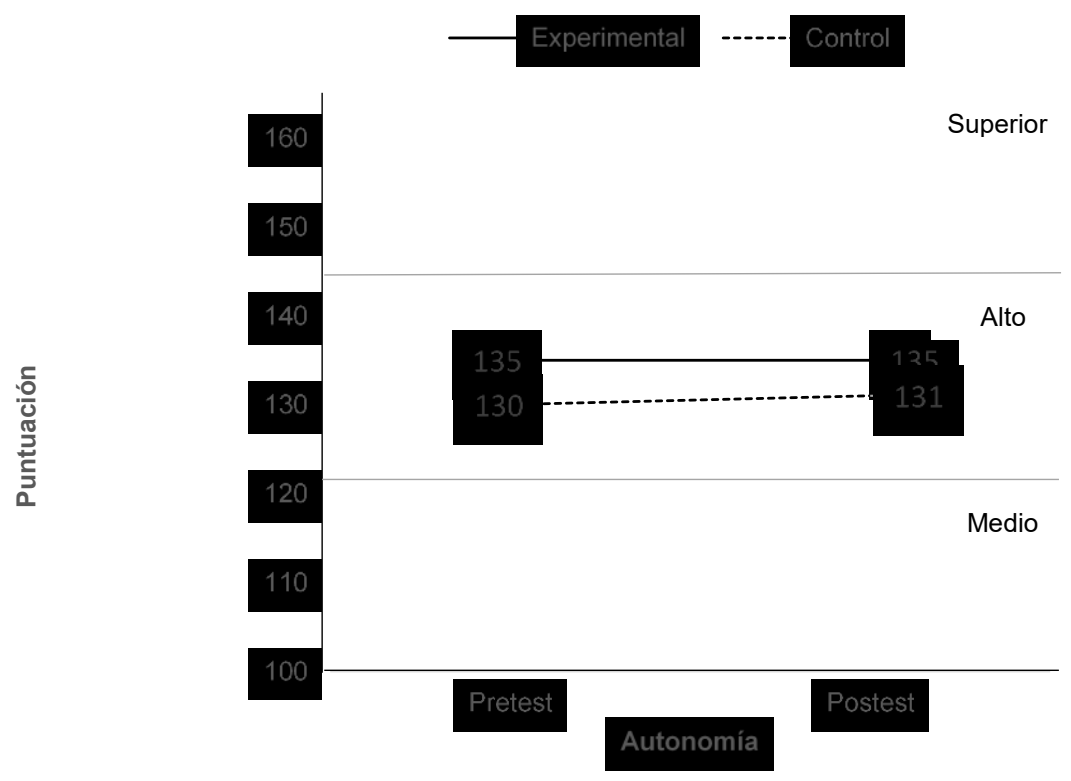

Figura 2. Resultado de media autonomía del grupo experimental y control

Ante la diferencia de medias, se decidió realizar la prueba de t-student (Tabla I). Para ello se comprobaron los supuestos de normalidad y equivalencia entre grupos, lo cual permitió asegurar la normalidad y la homogeneidad de varianzas. Posteriormente, se aplicó la t-student de muestras independientes entre el pre-test y el post-test de ambos grupos.

Tabla I.Pruebas $t$ student de muestras independientes: Desarrollo de la autonomía en grupo control y grupo experimental

\begin{tabular}{|c|c|c|c|c|c|c|c|c|c|}
\hline & \multirow[t]{2}{*}{ Grupo } & \multirow[t]{2}{*}{ Media } & \multirow[t]{2}{*}{$\begin{array}{l}\text { Desv. } \\
\text { Típica }\end{array}$} & \multirow{2}{*}{$\begin{array}{c}\text { Error } \\
\text { tip. } \\
\text { de la } \\
\text { media }\end{array}$} & \multicolumn{2}{|c|}{$\begin{array}{l}95 \% \text { Intervalo de } \\
\text { confianza para la } \\
\text { diferencia }\end{array}$} & \multirow[t]{2}{*}{$\mathbf{t}$} & \multirow[t]{2}{*}{ gl } & \multirow[t]{2}{*}{$\begin{array}{l}\text { Sig. } \\
\text { (bil.) }\end{array}$} \\
\hline & & & & & Inferior & Superior & & & \\
\hline \multirow{2}{*}{$\begin{array}{c}\text { AUTONOMIA } \\
\text { (Pre-test) }\end{array}$} & Experimental & 135,7931 & 8,88168 & 1,64929 & \multirow{2}{*}{$-1,28129$} & \multirow{2}{*}{11,4675} & \multirow{2}{*}{1,6} & \multirow{2}{*}{57} & \multirow{2}{*}{0,115} \\
\hline & Control & 130,7 & 14,74882 & 2,69275 & & & & & \\
\hline \multirow{2}{*}{$\begin{array}{c}\text { AUTONOMIA } \\
\text { (Post-test) }\end{array}$} & Experimental & 135,4483 & 9,91963 & 1,84203 & \multirow{2}{*}{$-1,33515$} & \multirow{2}{*}{8,89836} & \multirow{2}{*}{1,48} & \multirow{2}{*}{57} & \multirow{2}{*}{0,144} \\
\hline & Control & 131,6667 & 9,70721 & 1,77229 & & & & & \\
\hline
\end{tabular}

En cuanto al pre-test, no se encontraron diferencias estadísticamente significativas en el desarrollo de la autonomía entre el grupo experimental y el grupo control $(t=1,6 ; p=0,11)$. Y en relación al post-test, tampoco se encontraron diferencias estadísticamente significativas en el desarrollo de la autonomía entre el grupo experimental y el grupo control $(t=1,48 ; p=0.14)$. Lo 
anterior permite observar que la propuesta metodológica del ABP diseñada no permitió desarrollar la autonomía intelectual.

\section{Rúbrica de evaluación del Trabajo Colaborativo}

Se realizó una gráfica descriptiva exponencial que describe los resultados finales de las rúbricas, encontrando que la metodología $\mathrm{ABP}$ promovió el desarrollo del trabajo colaborativo en los estudiantes del grupo experimental del CCH-Vallejo.

En la Figura 3 se observa el crecimiento del trabajo colaborativo de los cinco grupos de trabajo del aula a medida que avanzaban las sesiones. Se graficó una línea de tendencia que permite ver que los puntajes ascienden con el desarrollo de la materia y la construcción de conocimiento.

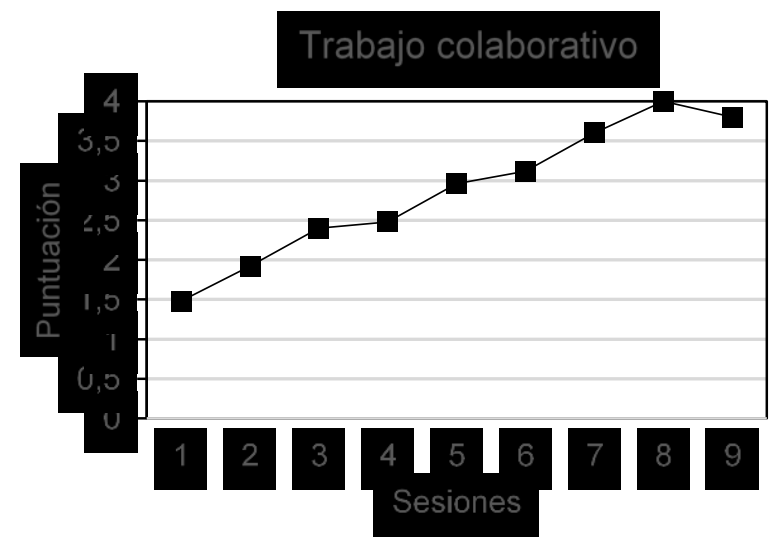

Figura 3. Desarrollo del trabajo colaborativo del grupo experimental

Para identificar la significancia estadística se realizó la prueba de Shapiro-Wilk, encontrando que las significancias eran menos a $(0,05)$, lo cual permitió afirmar que la distribución no era normal. Por ello se realizó la prueba no paramétrica de Wilcoxon, encontrando $(\mathrm{z}=-4,756 ; p=0,000)$, con esto se afirma que se encuentra evidencia suficiente para decir que la metodología ABP, como se observa en la Figura 4, produce un efecto diferencial en el trabajo colaborativo de los estudiantes con un nivel de confianza del 99,9\%. 


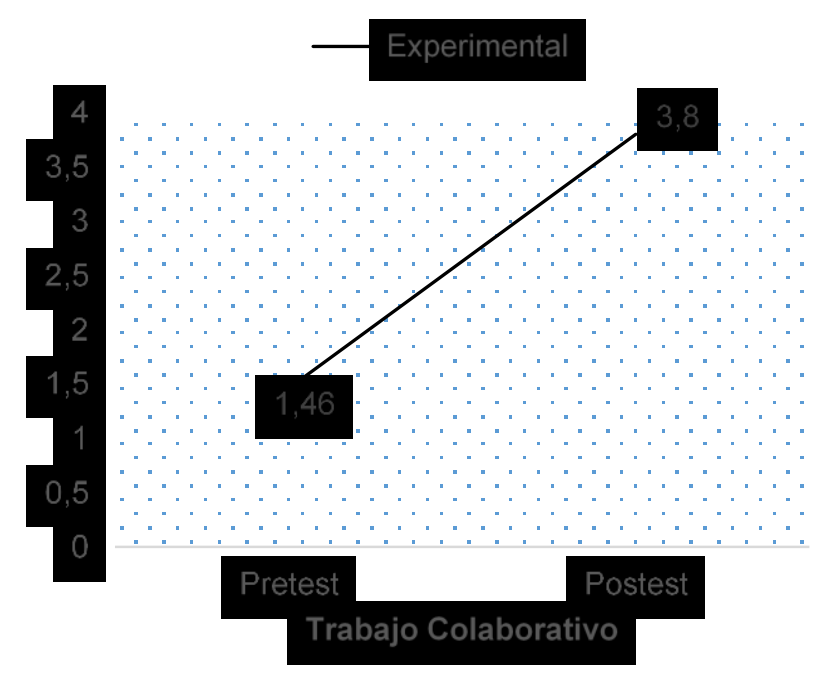

Figura 4. Media de Trabajo colaborativo del grupo experimental

\section{Cuestionario de Conocimientos sobre Diversidad de la Psicología}

Se aplicó la prueba estadística $t$-student de muestras independientes para identificar si existían diferencias estadísticamente significativas en el Cuestionario de conocimientos entre el pre-test y el post-test del grupo experimental y del grupo control. Lo anterior permite conocer si la metodología ABP implementada en la investigación promueve el mejor desempeño académico en el tema La diversidad de la psicología.

Según la Figura 5, producto de los análisis descriptivos, se evidencia que los dos grupos aumentaron su puntuación en el Cuestionario de conocimientos después de la intervención. También se puede concluir que los dos grupos presentan diferencias en el pre-test y el post-test; pues los estudiantes del grupo control poseen más conocimiento previo que los estudiantes del grupo experimental, y los estudiantes del grupo experimental poseen mayor conocimiento después de la intervención. Sin embargo, los dos grupos presentan un aumento en la construcción de conocimientos después de la intervención. 


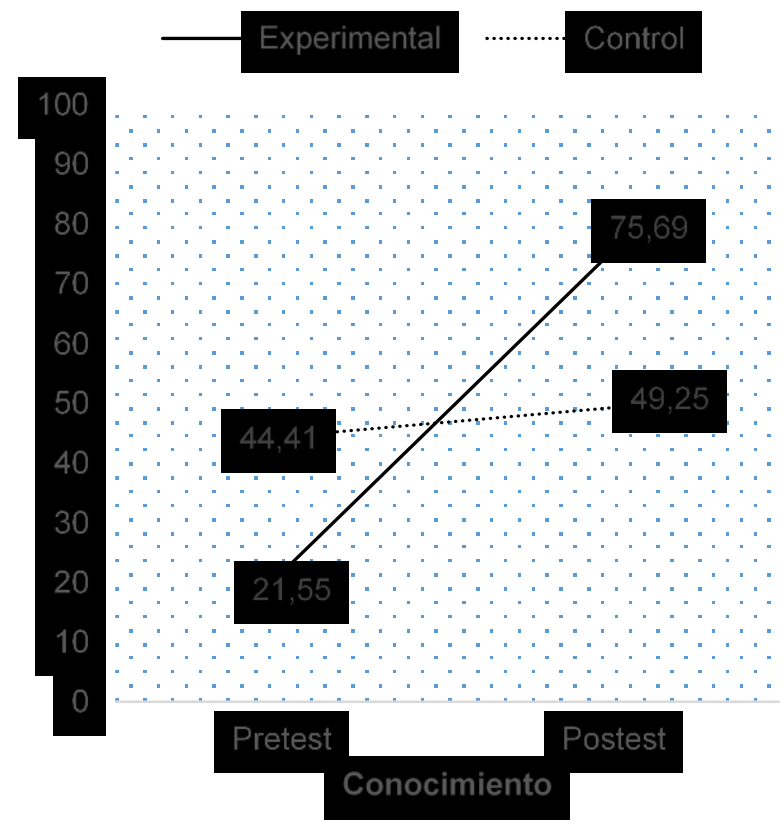

Figura 5. Comparación de los Descriptivos del Cuestionario de conocimientos

Como se observa en la Tabla II, no se encontraron diferencias estadísticamente significativas en el pre-test entre el grupo experimental y el grupo control $(t=-5,46 ; p=0.06)$. Lo anterior permite afirmar que estadísticamente los grupos poseían condiciones similares de conocimiento y fue posible compararlos. Por otro lado, para el post-test sí se encontraron diferencias estadísticamente significativas en la construcción de conocimientos de los estudiantes entre el grupo experimental y el grupo control $(t=5,6 ; \boldsymbol{p}=\mathbf{0 . 0 0})$. Lo cual permite inferir que la construcción de conocimientos fue mayor en el grupo experimental respecto al grupo control, y que la intervención educativa influyó en estos resultados.

Tabla II. Pruebas $t$ student de muestras independientes: Cuestionario de conocimientos en el grupo control y grupo experimental.

\begin{tabular}{|c|c|c|c|c|c|c|c|c|c|}
\hline & \multirow[t]{2}{*}{ Grupo } & \multirow[t]{2}{*}{ Media } & \multirow[t]{2}{*}{$\begin{array}{l}\text { Desv. } \\
\text { Típica }\end{array}$} & \multirow{2}{*}{$\begin{array}{c}\text { Error tip. } \\
\text { de la } \\
\text { media }\end{array}$} & \multicolumn{2}{|c|}{$\begin{array}{l}95 \% \text { Intervalo de } \\
\text { confianza para la } \\
\text { diferencia }\end{array}$} & \multirow[t]{2}{*}{$\mathbf{t}$} & \multirow[t]{2}{*}{ gl } & \multirow[t]{2}{*}{$\begin{array}{l}\text { Sig. } \\
\text { (bil.) }\end{array}$} \\
\hline & & & & & Inferior & Superior & & & \\
\hline \multirow{2}{*}{$\begin{array}{l}\text { CONOCIMIENTO } \\
\text { (Pre-test) }\end{array}$} & Experimental & 21,552 & 13,0831 & 2,4295 & \multirow{2}{*}{$-31,2397$} & \multirow{2}{*}{$-14,4902$} & \multirow{2}{*}{$-5,467$} & \multirow{2}{*}{57} & \multirow{2}{*}{0,06} \\
\hline & Control & 44,417 & 18,4844 & 3,3748 & & & & & \\
\hline \multirow{2}{*}{$\begin{array}{l}\text { CONOCIMIENTO } \\
\text { (Post-test) }\end{array}$} & Experimental & 75,69 & 15,824 & 2,9385 & \multirow{2}{*}{17,1246} & \multirow{2}{*}{35,7548} & \multirow{2}{*}{5,684} & \multirow{2}{*}{57} & \multirow{2}{*}{0,00} \\
\hline & Control & 49,25 & 19,6318 & 3,5843 & & & & & \\
\hline
\end{tabular}

Para identificar específicamente el comportamiento de los resultados del post-test de cada grupo, comparados con su propia línea base, se aplicó t-student de muestras relacionadas. 


\section{Grupo control}

De acuerdo a la Tabla III, las puntuaciones del Cuestionario de conocimientos no presentan diferencias estadísticamente significativas después de las nueve sesiones de trabajar el tema La diversidad de la psicología desde la propuesta de la profesora del grupo control $(t=1.01 ; p=0.32)$. Para este cálculo se tuvo en cuenta la distribución de grados de libertad que correspondía a n-1 (29), obteniendo un valor de 2,045 y al ser menor el resultado de la t, se encontró que las puntuaciones del test de conocimiento no se vieron afectadas después de las nueve sesiones de psicología.

Tabla III. Pruebas $t$ student de muestras relacionadas: Cuestionario de conocimientos en el grupo control

\begin{tabular}{|c|c|c|c|c|c|c|c|c|c|}
\hline & & \multirow[t]{2}{*}{ Media } & \multirow[t]{2}{*}{$\begin{array}{c}\text { Desv. } \\
\text { Típica }\end{array}$} & \multirow[t]{2}{*}{$\begin{array}{l}\text { Error tip. } \\
\text { de la media }\end{array}$} & \multicolumn{2}{|c|}{$\begin{array}{l}95 \% \text { Intervalo de } \\
\text { confianza para la } \\
\text { diferencia }\end{array}$} & \multirow[t]{2}{*}{$\mathbf{t}$} & \multirow[t]{2}{*}{ gl } & \multirow[t]{2}{*}{$\begin{array}{l}\text { Sig. } \\
\text { (bil.) }\end{array}$} \\
\hline & & & & & Inferior & Superior & & & \\
\hline Par 1 & $\begin{array}{c}\text { CONOCIMIENTO } \\
\text { (Pre-test) } \\
\text { CONOCIMIENTO } \\
\text { (Post-test) }\end{array}$ & $-4,8333$ & 26,1456 & 4,7735 & $-14,5963$ & 4,9296 & $-1,013$ & 29 & 0,32 \\
\hline
\end{tabular}

\section{Grupo experimental}

En los resultados que se muestran en la Tabla IV, se observa que las puntuaciones del Cuestionario de conocimientos presentan diferencias estadísticamente significativas después de las nueve sesiones de trabajar el tema La diversidad de la psicología con una propuesta desde la metodología ABP $(t=13.07 ; \boldsymbol{p}=\mathbf{0 . 0 0 0})$. Para este cálculo se tuvo en cuenta la distribución de grados de libertad que correspondía a n-1 (28), obteniendo un valor de 2,048 y al ser mayor el resultado de la t mayor. Se concluye que las puntuaciones del Cuesionario de conocimientos aumentaron significativamente después de la intervención con metodología ABP. 


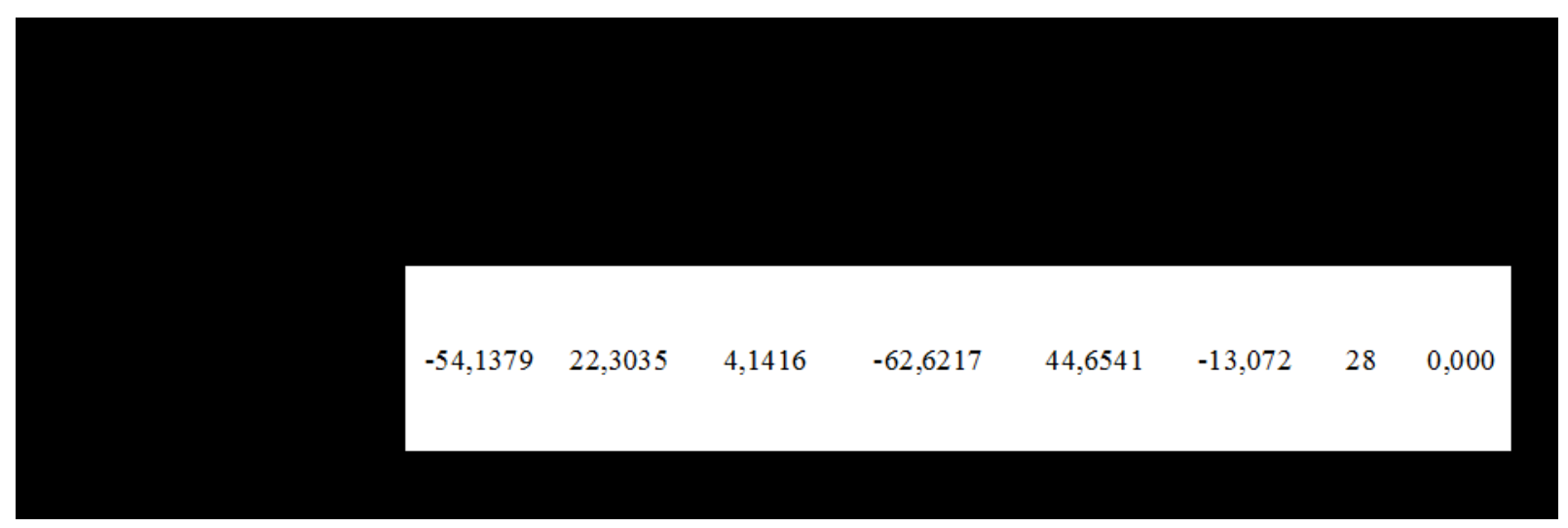

\section{Discusión}

Como parte del objetivo general, se buscaba promover la autonomía por medio de la metodología ABP. Después de la intervención, los estadísticos descriptivos sugirieron la promoción de la autonomía en el grupo experimental. Sin embargo los resultados no fueron estadísticamente significativos.

Por otro lado, Littlewood (1999), contemplando lo propuesto por Benson propone la existencia de la autonomía intelectual reactiva y proactiva. La reactiva referida a que el estudiante no determina sus orientaciones pues éstas son establecidas por el docente o la institución, pero organiza sus recursos de forma autónoma. Y la proactiva que permite al estudiante generar reflexiones sobre su proceso de aprendizaje, definir objetivos con la guía del docente, seleccionar rutas de aprendizaje y autoevaluar sus resultados en el proceso de construcción del conocimiento.

En esta investigación se pretendía desarrollar la autonomía intelectual ligada a aspectos proactivos. Según los resultados, no se encontraron diferencias significativas en la autonomía después de la intervención, es posible que esto se deba a que los estudiantes del $\mathrm{CCH}$, dentro del proceso educativo, no han tenido la necesidad de responsabilizarse y tener control sobre su propio aprendizaje; lo cual los sitúa en la autonomía intelectual reactiva.

Esta autonomía intelectual proactiva, según Benson (2001), se desarrolla con el tiempo y a la par con los procesos de desarrollo. Sin embargo, es necesario que el docente promueva al aprendiz para que desarrolle responsabilidad sobre su propio proceso de aprendizaje, guiándolo en su proceso de empoderamiento y aplicando estrategias metacognitivas, sociales y afectivas.

La promoción del trabajo colaborativo que se observó como resultado de esta investigación, abona evidencia que se suma a las investigaciones de González, Martín, Carbonero y Lara (2013); Pantoja y Covarrubias (2013) quienes señalan que mediante el ABP 
los estudiantes perciben una promoción de la colaboración al tomar decisiones en equipo, ayudarse, conocerse y retroalimentarse mutuamente.

Se atribuye el éxito de la intervención a la realización de actividades en donde los estudiantes tuvieron un objetivo común de grupo y que cada uno aportó un elemento para alcanzar la meta propuesta, ratificando que dentro del desarrollo de las estrategias de aprendizaje contempladas en la construcción del diseño instruccional y la intervención, se debe tener presente la importancia de la comunicación, conversación y el acceso a la información (Kauchak y Eggen, 1998).

Por otro lado, al igual que Im y Lee (2003), en esta investigación se considera que la retroalimentación oportuna a los estudiantes por parte del docente y sus pares, permite promover la comunicación por medio de discusiones sincrónicas y asincrónicas; desde la interacción social hasta el desarrollo de la tarea.

En el pre-test, se observaron diferencias en los conocimientos previos para el tema de la diversidad de la psicología, ya que el grupo control tenía una puntuación mayor que el grupo experimental.

Sin embargo, al realizar las pruebas estadísticas no se encontraron diferencias significativas entre el pre-test y el post-test del grupo control, por lo que es posible suponer que el rendimiento se ve afectado -disminuido- si las actividades planeadas por el docente no implican un reto para el estudiante.

El hallazgo anterior contradice la afirmación sobre el conocimiento previo como predictor del buen rendimiento futuro del estudiante (Escudero, 1981; Reparaz, Tourón y Villanueva, 1990).

Por otro lado, Perez y Díaz (1994) en sus investigaciones sobre niños superdotados y su bajo rendimiento en escuelas regulares, argumentan que aunque existe conocimiento previo, la baja dificultad de la tarea y la desmotivación de los estudiantes dificultan su rendimiento futuro.

En esta investigación se encuentra algo similar, ya que los resultados del post-test no indican un mejor desempeño en aquellos estudiantes con mayores conocimientos previos, sino en los que fueron intervenidos con metodología ABP. Por lo que es posible suponer que cuando existen conocimientos previos sobre un tema, se deben utilizar mayores estrategias de motivación que lleven al estudiante a utilizar sus potencialidades para co-construir el conocimiento que ya han adquirido (Jiménez, Vega, Capa, Fierro y Quichimbo, 2019). 
En esto radica la importancia de la evaluación diagnóstica, si se reconoce al estudiante como un sujeto activo, el docente debería identificar los conocimientos previos e intereses de éste al inicio de cualquier curso.

En cuanto al grupo experimental, si se encontraron diferencias significativas entre pretest y post-test. Este resultado puede ser explicado según Morales y Landa (2008) como una de las bondades del ABP, puesto que desde el inicio del proceso de la aplicación de la estrategia permite explorar los aprendizajes previos de los estudiantes y contemplarlos dentro de las sesiones.

Al igual que Barrows (1986); Urrutia, Hamui-Sutton, Castañeda, Fortoul van der Goes y Guevara (2011); Restrepo et al. (1997) y Romero, Rodríguez y Gómez (2008), en este trabajo la metodología ABP también mejoró la comprensión y la construcción de conocimientos de los estudiantes motivándolos a investigar y aprender, incluso cuando fue llevado a cabo en un nivel educativo medio superior.

Aún y cuando los resultados de la presente investigación coinciden con múltiples estudios, difieren de los resultados de otras investigaciones que no hallaron diferencias significativas en el aprendizaje de contenidos empleando ABP (Fasce et al., 2001; Fuentes y Pérez, 2013). Es probable que en estas investigaciones se hayan empleado algunos elementos del ABP, como es la utilización de un problema, pero no se hayan incorporado otros elementos que también son importantes en la metodología, como serían, los espacios de aprendizaje autónomo, los espacios de socialización del conocimiento, los roles dentro del grupo, el papel facilitador del docente, el rol activo del estudiante y la planeación microcurricular.

A manera de cierre, el trabajo colaborativo, implícito en la metodología ABP, permite que se genere la colaboración entre miembros del grupo con mayores conocimientos previos; actuando éstos a su vez como facilitadores de sus pares en el proceso de construcción del conocimiento y generando procesos de andamiaje. Lo anterior permite que todos los actores construyan los conocimientos esperados y, de acuerdo con sus experiencias previas, elaboren nuevos conocimientos que los guíen a un estadio superior al esperado.

Se puede atribuir el éxito de la intervención al proceso de construcción del diseño microcurricular desde la metodología ABP, al proceso realizado teniendo en cuenta los fundamentos metodológicos en la asignatura La diversidad de la psicología, y a la utilización de material didáctico digital (TIC) desde la perspectiva del cambio social, en donde la 
metodología y las actividades de la planeación promueven el aprendizaje de los estudiantes (Castellano, Luque y Pantoja, 2017; Bloome, Castanheira, Leung y Rowsell, 2019).

Adicionalmente, la utilización de la plataforma virtual permitió complementar el proceso de enseñanza-aprendizaje, ya que los estudiantes accedieron a material didáctico complementario como actividades audiovisuales e interactivas, y el docente pudo observar el desempeño individual y reflexionar sobre los procesos de construcción del conocimiento de los estudiantes a lo largo de las sesiones.

Para futuras investigaciones que estén interesadas en utilizar la metodología ABP, se recomienda considerar todos los elementos descritos en esta investigación, incluyendo el uso e implementación de las TIC' s, y no priorizar sólo alguno de ellos como la utilización de un problema. Adicionalmente, se sugiere explorar la metodología ABP en diferentes niveles educativos y contextos diversos, e incluir planeaciones de asignaturas en nivel medio superior que se realicen con base en una metodología $\mathrm{ABP}$, en particular, en el $\mathrm{CCH}$, ya que permite aumentar el desempeño académico, mitigar las tasas de reprobación, la baja eficiencia terminal, cumplir con los objetivos institucionales y preparar al adolescente para el próximo nivel de estudios y para la vida.

\section{Referencias}

Albanese, M., y Mitchell, S. (1993). Problem-Based Learning: A reviewof the literatura, its outcomes and implementation issues. Academic Medicine. 68(1), 52- 81. https://pubmed.ncbi.nlm.nih.gov/8447896/

Álvarez A., y Del Río P. (1990). Educación y desarrollo: la teoría de Vigotsky y la zona de desarrollo próximo. En Coll, C., Palacios, J., y Marchesi, A. (Eds.) Desarrollo psicológico y educación. Psicología de la educación escolar. (1.a ed., vol. 2). Madrid: Alianza.

Ausubel, D., Novak, J., y Hanesian, H. (2012). Psicología educativa. Un punto de vista cognoscitivo (2. ${ }^{\mathrm{a}}$ ed.). México: Trillas. (Original publicado en 1983)

Barrows, H. (1986). A Taxonomy of problem based learning methods. Medical Education, 20, 481-486.

Barrows, H. (1992). The Tutorial Process. Springfield: Southern Illinois University School of Medicine.

Benítez, M., Cruces, E., De Haro, J. y Sarrión, M. (2010, 8-9 de julio). Aprendizaje basado en problemas a través de las TIC [ponencia]. XIX Jornadas de la Asociación de Economía de la Educación, Zaragoza, España.

https://2010.economicsofeducation.com/es/program/index.html 
Benson, P. (2001). Teaching and Researching Autonomy in Language Learning. Harlow: Pearson Education Limited.

Biggs, J. (2010). Calidad del aprendizaje universitario (Teaching for quality learning at university, trad.). Madrid: Narcea. https://barajasvictor.files.wordpress.com/2014/05/libro-j-biggs.pdf

Bloome, D., Castanheira, M., Leung, C., y Rowsell, J. (eds). (2019). Re-theorizing literacy practices. Complex Social and Cultural Contexts. Routledge. https://www.taylorfrancis.com/books/e/9781351254229

Boud, D., y Feletti, G. (1997). The challenge of problem-based learning (2a ed.). London: Kogan Page Limited. https://eric.ed.gov/?id=ED415220 (Original publicado en 1991).

Branda, L. A. (2009). El aprendizaje basado en problemas. De herejía artificial a res popularis. Educación Médica, 12(1), 11-23.

Brockbank, A. y McGill, I. (2002). Aprendizaje reflexivo en la educación superior. Morata. https://edmorata.es/libros/el-curriculum-oculto/

Bruner, J. (1971). The relevance of education. New York: Norton.

Carretero, M. (2009). Constructivismo y educación. Paidós.

Castellano, E. y Pantoja, A. (2017). Eficacia de un programa de intervención basado en el uso de las TIC en la tutoría. Revista de Investigación Educativa, 35(1), 215-233. DOI:http://dx.doi.org/10.6018/rie.35.1.248831

Clemens, A., Tapia, S., y Olivares, S. (2015). Desarrollo del pensamiento crítico mediante el aprendizaje colaborativo en alumnos de primaria. Rieege. 6 (11), 1-57.

Coll, C., Palacios, J., y Marchesi, A. (Compiladores) (2001). Desarrollo psicológico y educación (1 ${ }^{\mathrm{a}}$ ed., vol. 2). Psicología de la educación escolar. Madrid: Alianza.

Cyr, P. (1996). Les stratégies d'apprentissage d'une langue seconde (1 ${ }^{\mathrm{a}} \mathrm{ed}$.). Anjou [Québec]: Editions CEC.

Díaz-Barriga, F. (2005). Enseñanza situada: Vínculo entre la escuela y la vida (1ª ed.). México: McGraw Hill.

Díaz Barriga, F. y Hernández, G. (2002). Constructivismo y aprendizaje significativo. En: Estrategias docentes para un aprendizaje significativo (2 ${ }^{\mathrm{a}}$ ed.). México: Mc Graw Hill. (Original publicado en 1999).

Duch, B., Groh, S., y Allen, D. (2001). The power of problem-based learning (1 $\left.{ }^{\mathrm{a}} \mathrm{ed}.\right)$. Virginia: Stylus Publishing; LLC.

Dueñas, V. H. (2001): El aprendizaje basado en problemas como enfoque pedagógico en la educación en salud. Colomb. Medica, 32(4) 189-196. http://colombiamedica.univalle.edu.co/Vol32No4/aprendizaje.pdf.

ENCCH. (2017). Informe de gestión 2017. http://www.planeacion.unam.mx/informes/PDF/CCH-2017.pdf 
ENDEMS. (2012). Reporte de la Encuesta Nacional de Deserción en la Educación Media Superior.

http://www.sems.gob.mx/work/models/sems/Resource/10787/1/images/Anexo 6Repor te de la ENDEMS.pdf.

Escudero, J. M. (1981). Modelos Didácticos (1ª ed.). Barcelona: Oikos Tau.

Fasce, H., Calderón, B., Braga, I. De Orúe, M., Mayer, H., Wagemann, H., y Cid, S. (2001). Utilización del aprendizaje basado en problemas en la enseñanza de física en estudiantes de medicina. Comparación con enseñanza tradicional. Revista Medica de Chile, 129(9), 103-137. http://dx.doi.org/10.4067/S0034-98872001000900008

Flores, L., y Meléndez, C. (2017). Variación de la autonomía en el aprendizaje, en función de la gestión del conocimiento, para disminuir en los alumnos los efectos del aislamiento. Revista de Educación a Distancia (RED), 17(54), 1-15. https://revistas.um.es/red/article/view/298871

Fuentes, V., y Pérez, C. (2013). Estudio comparativo entre metodologías Aprendizaje Basado en Problemas y tradicional en Módulo de Enseñanza. Revista de Educación en Ciencias de la Salud, 10(2) 107-113. http://www2.udec.cl/ofem/recs/

González, G., Martín, P., Carbonero, M., y Lara, F. (2013). Evaluación por competencias de los estudiantes de Enfermería a través del Aprendizaje Basado en Problemas. Enfermería Universitaria. 10(4), 120-124. https://doi.org/10.1016/S1665-7063(13)72640-X

Gómez, B., y Mei, P. (2007). Promoting positive young development in schools. Theory into Practice. 46(2), 97-104. https://doi.org/10.1080/00405840701232752

Hmelo-Silver, C. E., y Ferrari, M. (1997). The Problem-Based Learning tutorial: Cultivating Higher Order Thinking Skills. Journal for the Education of the Gifted, 20(4), 401-422. https://doi.org/10.1177\%2F016235329702000405

Im, Y., y Lee, O. (2003). Pedagogical Implications of Online Discussion for Preservice Teacher. Training. Journal of Research On Technology In Education, 36(2), 155-170. https://doi.org/10.1080/15391523.2003.10782410

Jiménez, L. S., Vega, N., Capa, E. D., Fierro, N. del C. y Quichimbo, P. (2019). Estilos y estrategia de enseñanza-aprendizaje de estudiantes universitarios de la Ciencia del Suelo. Revista Electrónica de Investigación Educativa (REDIE), 21(4), 1-10. https://doi.org/10.24320/redie.2019.21.e04.1935

Kauchak, D., y Eggen, P. (1998). Learning \& teaching: Research-based methods (3rd ed.). Boston: Allyn y Bacon. (Original publicado en 1989).

Kolloffel, B., Eysink, T. y Jong, T. (2011). Comparing the Effects of Representational Tools in Collaborative and Individual Inquiry Learning. Computer-Supported Collaborative Learning, 6, 223-251. https://doi.org/10.1007/s11412-011-9110-3

Lai, P., y Chu, K. (2000, 2-5 July). Can PBL work for surface students? [conference]. ASET / HERDSA Joint International Conference. Queensland, Australia. https://www.ascilite.org/conferences/aset-archives/confs/asetherdsa2000/procs/chu1.html 
Littlewood, W. (1999). Defining and Developing Autonomy in East Asian Contexts. Applied Linguistics, 20(1), 71-94. https://doi.org/10.1093/applin/20.1.71

Martínez, V. (2009). Diversos condicionantes del fracaso escolar en la educación secundaria. Revista Iberoamericana de Educación, 51, 67-85. https://doi.org/10.35362/rie510622

Mondragón, A., y Fuentes, H. (2016). La impartición de mejores clases podría contribuir a disminuir la deserción en el bachillerato. Poiética, (7), 28-33. http://www.cchnaucalpan.unam.mx/V2018/imgprin/publicaciones/Poietica/Poietica7.pdf

Morales, P., y Landa, V. (2004). Aprendizaje basado en problemas problem - based learning. Theoria, 13 ,

145-157. https://cmapspublic.ihmc.us/rid=1152868048651_1396346847_11204/pbl1.pdf

OCDE. (2014). Panorama de la educación 2014: Indicadores OCDE. http://www.oecd.org/education/Mexico-EAG2014-Country-Note-spanish.pdf

O’Malley, J. M., y Chamot, A. U. (1990). Learning strategies in second language acquisition (1. ${ }^{\text {st }}$ ed.). Cambridge, UK: Cambridge University Press.

Oxford, R. (2011). Teaching and Researching: Language Learning Strategies (1. ${ }^{\text {st }}$ ed.). Edimburg Gate: Pearson Education Limited, (342 p.)

Pantoja, J., y Covarrubias, P. (2013). La enseñanza de la biología en el bachillerato a partir del aprendizaje basado en problemas (ABP). Perfiles Educativos, 32(139), 93-109. https://doi.org/10.22201/iisue.24486167e.2013.139.35714

Perez, L y Diaz, O. (1994). Bajo rendimiento académico y desintegración. Faisca, Revista altas capacidades, 1, 103-128. https://dialnet.unirioja.es/servlet/articulo? codigo $=2477692$

Prieto, A., Barbarroja, J., Reyes, E., Monserrat, J., Diaz, D., Villarroel, M., y Álvarez, M. (2006). Un nuevo modelo de aprendizaje basado en problemas, el ABP 4x4, es eficaz para desarrollar competencias profesionales valiosas en asignaturas con más de 100 alumnos. Aula abierta, 87, 171-194. http://www.journals4free.com/link.jsp?1=38964792

Repáraz, C., Tourón, J., y Villanueva, C. (1990). Estudio de algunos factores relacionados con el éxito académico en $8^{\circ}$ de EGB. Bordón, 42(2), 167-178. https://dialnet.unirioja.es/servlet/articulo? codigo $=54333$

Restrepo, B., García, N., Ceballos, M., Arango. A., Aponte, A., Bernal, T. Ramírez, L., Gómez, M., Yepez, T., Calvo, Y., Beltrán, H., Sierra, J., Escobar, J., y Velasquez, A. (1997). El aprendizaje basado en problemas en la formación de profesionales de la salud, RionegroAntioquia: 1996. Investigación y Educación en Enfermería, 15(2), 83-103. https://revistas.udea.edu.co/index.php/iee/article/view/16946/14677

Ríos, L., Urrutia, M., y Contreras, O. (2013). Moléculas presentes en las células: un logro de los aprendizajes con la estrategia de ABP. Didáctica en las ciencias. https://www.raco.cat/index.php/Ensenanza/article/viewFile/308186/398180

Romero, J., Rodríguez, A., y Gómez, J. (2008). Evaluación de escenarios para el aprendizaje basado en problemas (ABP) en la asignatura de química de bachillerato. Didáctica de la química. Educación química, 19(3) http://www.scielo.org.mx/scielo.php?pid=S0187$\underline{893 \times 2008000300006 \& \text { script }=\text { sci arttext }}$ 
Romero, A., Jara, P., Marín, F., Millán, A. y Carrillo, E. (2009, 22 de junio). El aprendizaje basado en problemas (ABP) como práctica multiasignatura con grupos grandes. Experiencia de una práctica ABP en cuatro asignaturas de primer curso de licenciado en Psicología de la Universidad de Murcia. Taller international sobre ABP/EBL. International Workshop on Problem Based Learning and Enquired Based Learning. Madrid. $\quad$ https://www.um.es/docencia/agustinr/ie/prodcien/13-2009PosterABPprimero6-UAM.pdf

Rué, J. (2009). El Aprendizaje Autónomo en Educación Superior. España: Narcea.

Schmidt, H. G. (1983). Problem-Based Learning: Rationale and Description. Medical Education, 17, 11-16. https://doi.org/10.1111/j.1365-2923.1983.tb01086.x

Sola, C. (2005). Aprendizaje Basado en Problemas, de la teoría a la práctica. México: Trillas.

Solórzano-Mendoza, Y. (2017). Aprendizaje autónomo y competencias. Dominio de las Ciencias, $3, \quad$ 241-253. https://dominiodelasciencias.com/ojs/index.php/es/article/view/390/pdf

Urrutia, M., Hamui-Sutton, A., Castañeda, S., Fortoul van der Goes, T., y Guevara, R. (2011). Impacto del aprendizaje basado en problemas en los procesos cognitivos de los estudiantes de medicina. Gaceta Médica de México. 147, 385-393. http://anmm.org.mx/GMM/2011/n5/14 GMM Vol 147 - 5 2011.pdf

Vizcarro, C., y Juárez, E. (2008). ¿Qué es y cómo funciona el aprendizaje basado en problemas?. En García, J. (Ed.). El aprendizaje basado en problemas en la enseñanza universitaria (pp. 17-36). España: Editorial Universidad de Murcia.

Wilkerson, L., y Gijselaers, W. (1996). Bringing problem-based learning to higher education: theory and practice. San Francisco: Jossey-Bass Inc. Publishers.

William, S., y Gallagher, S. (Eds.). (1993). Problem-based learning: As authentic as it gets. Educational Leadership, 50(7), 25-28. http://www.ascd.org/publications/educational_leadership/apr93/vol50/num07/ProblemBased_Learning@_As_Authentic_As_It_Gets.aspx

Autoras Angie Ximena Valles Ramírez. https://orcid.org/0000-0002-5159-2806

Universidad Nacional Autónoma de México (UNAM), México. Maestra en Docencia para la Educación Media Superior por la UNAM. Psicóloga por la Fundación Universitaria Sanitas. Equipo editorial de la Revista Internacional de Psicología.

Patricia Covarrubias-Papahiu. https://orcid.org/0000-0001-9855-3399 Universidad Nacional Autónoma de México (UNAM), México. Dra. en Pedagogía, y psicóloga por la Universidad Nacional Autónoma de México (UNAM). Profesora Titular C e investigadora de la Carrera de psicología, de la Facultad de Estudios Superiores Iztacala (UNAM) 